\title{
A Modified Abramov-Petkovšek Reduction and Creative Telescoping for Hypergeometric Terms
}

\author{
Shaoshi Chen ${ }^{1}$, Hui Huangg, ${ }^{1,2}$ Manuel Kauers ${ }^{2}$, Ziming $\mathrm{Li}^{1}$ \\ ${ }^{1} \mathrm{KLMM}$, AMSS, Chinese Academy of Sciences, Beijing 100190, (China) \\ ${ }^{2}$ RISC, Johannes Kepler University, Linz A-4040, (Austria) \\ schen, huanghui@amss.ac.cn \\ mkauers@risc.jku.at, zmli@mmrc.iss.ac.cn
}

\begin{abstract}
The Abramov-Petkovšek reduction computes an additive decomposition of a hypergeometric term, which extends the functionality of the Gosper algorithm for indefinite hypergeometric summation. We modify the AbramovPetkovšek reduction so as to decompose a hypergeometric term as the sum of a summable term and a non-summable one. The outputs of the Abramov-Petkovšek reduction and our modified version share the same required properties. The modified reduction does not solve any auxiliary linear difference equation explicitly. It is also more efficient than the original reduction according to computational experiments. Based on this reduction, we design a new algorithm to compute minimal telescopers for bivariate hypergeometric terms. The new algorithm can avoid the costly computation of certificates.
\end{abstract}

\section{Categories and Subject Descriptors}

I.1.2 [Computing Methodologies]: Symbolic and Algebraic Manipulation-Algebraic Algorithms

\section{General Terms}

Algorithms, Theory

\section{Keywords}

Abramov-Petkovšek reduction, Hypergeometric term, Telescoper, Summability

\footnotetext{
${ }^{*}$ S. Chen was supported in part by the NSFC grant 11371143 and by President Fund of Academy of Mathematics and Systems Science, CAS (2014-cjrwlzx-chshsh), H. Huang by the Austrian Science Fund (FWF) grant W1214-13, M. Kauers by the Austrian Science Fund (FWF) grants Y464-N18 and F50-04, H. Huang and Z. Li by two NSFC grants (91118001, 60821002/F02) and a 973 project (2011CB302401).

Permission to make digital or hard copies of all or part of this work for personal or classroom use is granted without fee provided that copies are not made or distributed for profit or commercial advantage and that copies bear this notice and the full citation on the first page. Copyrights for components of this work owned by others than ACM must be honored. Abstracting with credit is permitted. To copy otherwise, or republish, to post on servers or to redistribute to lists, requires prior specific permission and/or a fee. Request permissions from permissions@acm.org. ISSAC'15, July 6-9, 2015, Bath, United Kingdom.

Copyright is held by the owner/author(s). Publication rights licensed to ACM.

Copyright (C) 2015 ACM 978-1-4503-3435-8/15/07 ...\$15.00.

DOI: http://dx.doi.org/10.1145/2755996.2756648.
}

\section{INTRODUCTION}

Creative telescoping is a staple of symbolic summation. Its main use is to construct recurrence equations that have a prescribed definite sum among their solutions. By using other algorithms applicable to recurrence equations, it is then possible to derive interesting facts about the original definite sum, such as closed forms or asymptotic expansions.

The computational problem of creative telescoping is to construct, for a given term $f(x, y)$, polynomials $\ell_{0}, \ldots, \ell_{r}$ in $x$ only, not all zero, and another term $g(x, y)$ s.t.

$$
\ell_{0}(x) f(x, y)+\cdots+\ell_{r}(x) f(x+r, y)=g(x, y+1)-g(x, y) .
$$

The number $r$ may or may not be part of the input.

We can distinguish four generations of creative telescoping algorithms. The first generation was based on elimination techniques $[15,22,19,14]$. The second generation started with what is now known as Zeilberger's algorithm [21, $5,23,19]$. The algorithms of this generation use the idea of augmenting an algorithm for indefinite summation (or integration) by additional parameters $\ell_{0}, \ldots, \ell_{r}$ that are carried along during the calculation and are finally instantiated, if at all possible, such as to ensure the existence of a term $g$ as needed for the right-hand side. See [19] for details about the first two generations.

The third generation was initiated by Apagodu and Zeilberger $[17,6]$. In a sense, they applied a second-generation algorithm by hand to a generic input and worked out the resulting linear system of equations for the parameters $\ell_{i}$ and the coefficients inside the desired term $g$. Their algorithm then merely consists in solving this system. This approach is interesting not only because it is easier to implement and tends to run faster than earlier algorithms, but also because it is easy to analyze. In fact the analysis of algorithms from this family gives rise to the best output size estimates for creative telescoping known so far [11, 12, 13]. A disadvantage is that these algorithms may not always find the smallest possible output.

The fourth generation of creative telescoping algorithms originates from [7]. The basic idea behind these algorithms is to bring each term $f(x+i, y)$ of the left-hand side into some kind of normal forms modulo all terms that are differences of other terms. Then to find $\ell_{0}, \ldots, \ell_{r}$ amounts to finding a linear dependence among these normal forms. The key advantage of this approach is that it separates the computation of the $\ell_{i}$ from the computation of $g$. This is desirable in the typical situation where we are only 
interested in the $\ell_{i}$ and their size is much smaller than the size of $g$. With previous algorithms there was no way to obtain $\ell_{i}$ without also computing $g$, but with fourth generation algorithms there is. So far this approach has only been worked out for several instances in the differential case $[7,9,8]$. The goal of the present paper is to give a fourth-generation algorithm for the discrete case, namely for the classical setting of hypergeometric telescoping.

Our starting point is the Abramov-Petkovšek reduction for hypergeometric terms introduced in [3] and summarized in Section 3 below. Unfortunately the reduced forms obtained by this reduction are not sufficiently "normal" for our purpose. Therefore, in Sections 4 and 5 we present a refined variant of the reduction process and show that the corresponding normal forms are well-behaved with respect to taking linear combinations. Then in Section 6 we describe the creative telescoping algorithm obtained from this reduction. The final section contains an experimental comparison between this algorithm and the built-in algorithm of Maple.

\section{PRELIMINARIES}

Throughout the paper, we let $\mathbb{F}$ be a field of characteristic zero, and $\mathbb{F}(y)$ be the field of rational functions in $y$ over $\mathbb{F}$. Let $\sigma_{y}$ be the automorphism that maps $r(y)$ to $r(y+1)$ for every $r \in \mathbb{F}(y)$. The pair $\left(\mathbb{F}(y), \sigma_{y}\right)$ is called a difference field. A difference ring extension of $\left(\mathbb{F}(y), \sigma_{y}\right)$ is a ring $\mathbb{D}$ containing $\mathbb{F}(y)$ together with a distinguished endomorphism $\sigma_{y}: \mathbb{D} \rightarrow \mathbb{D}$ whose restriction to $\mathbb{F}(y)$ agrees with the automorphism defined before. An element $c \in \mathbb{D}$ is called a constant if $\sigma_{y}(c)=c$. For a nonzero polynomial $p \in \mathbb{F}[y]$, its degree and leading coefficient are denoted by $\operatorname{deg}_{y}(p)$ and $\operatorname{lc}_{y}(p)$, respectively.

Definition 2.1. Let $\mathbb{D}$ be a difference ring extension of $\mathbb{F}(y)$. A nonzero element $T \in \mathbb{D}$ is called a hypergeometric term over $\mathbb{F}(y)$ if $\sigma(T)=r T$ for some $r \in \mathbb{F}(y)$. We call $r$ the shift quotient of $T$ w.r.t. $y$.

The product of hypergeometric terms is again hypergeometric. Two hypergeometric terms $T_{1}, T_{2}$ are called similar if there exists a rational function $r \in \mathbb{F}(y)$ s.t. $T_{1}=r T_{2}$. By Proposition 5.6.2 in [19], the sum of similar hypergeometric terms is either hypergeometric or zero.

A univariate hypergeometric term $T$ is called hypergeometric summable if there exists another hypergeometric term $G$ s.t. $T=\Delta_{y}(G)$, where $\Delta_{y}$ denotes the difference of $\sigma_{y}$ and the identity map. We abbreviate "hypergeometric summable" as "summable" in this paper.

Given a hypergeometric term $T$, we let $\mathbb{U}_{T}$ be the union of $\{0\}$ and the set of summable hypergeometric terms that are similar to $T$. Then $\mathbb{U}_{T}$ is an $\mathbb{F}$-linear subspace of $\mathbb{D}$. Note that $\mathbb{U}_{T}=\mathbb{U}_{H}$ if $H$ is a hypergeometric term similar to $T$.

Recall $[3, \S 1]$ that a nonzero polynomial in $\mathbb{F}[y]$ is said to be shift-free if it is coprime with any of its nontrivial shifts. A nonzero rational function is said to be shift-reduced if its numerator is coprime with any shift of its denominator.

A basic property of shift-reduced rational functions is given below.

Lemma 2.2. Let $f \in \mathbb{F}(y)$ be shift-reduced and unequal to one. If there exists $r \in \mathbb{F}[y]$ s.t. $f \sigma_{y}(r)-r=0$, then $r=0$.

Proof. Suppose that $r \neq 0$. Then $f=r / \sigma_{y}(r)$. Since $f$ is unequal to one, $r$ does not belong to $\mathbb{F}$. It follows that $f$ is not shift-reduced, a contradiction.
According to $[3,4]$, every hypergeometric term $T$ has a multiplicative decomposition $S H$, where $S$ is in $\mathbb{F}(y)$ and $H$ is another hypergeometric term whose shift quotient is shiftreduced. We call the shift quotient $K:=\sigma_{y}(H) / H$ a kernel of $T$ w.r.t. $y$ and $S$ the corresponding shell. Note that $K=1$ if and only if $T$ is a rational function, which is then equal to $c S$ for some element $c \in \mathbb{D}$ with $\sigma_{y}(c)=c$.

Let $T=S H$ be a multiplicative decomposition, where $S$ is a rational function and $H$ a hypergeometric term with a kernel $K$. Assume that $T=\Delta_{y}(G)$ for some hypergeometric term $G$. A straightforward calculation shows that $G$ is similar to $T$. So there exists $r \in \mathbb{F}(y)$ s.t. $G=r H$. One can easily verify that

$$
S H=\Delta_{y}(r H) \Longleftrightarrow S=K \sigma_{y}(r)-r .
$$

Let $\mathbb{V}_{K}=\left\{K \sigma_{y}(r)-r \mid r \in \mathbb{F}(y)\right\}$, which is an $\mathbb{F}$-linear subspace of $\mathbb{F}(y)$. Then (1) translates into

$$
S H \equiv 0 \bmod \mathbb{U}_{H} \Longleftrightarrow S \equiv 0 \bmod \mathbb{V}_{K} .
$$

These congruences enable us to shorten expressions.

\section{ABRAMOV-PETKOVŠEK REDUCTION}

Reduction algorithms have been developed for computing additive decompositions of rational functions [1], hyperexponential functions [8], and hypergeometric terms [3, 4]. These algorithms can be viewed as generalizations of the Gosper algorithm [16, 19] and its differential analogue [5].

The Abramov-Petkovšek reduction [3, 4] is fundamental for this paper. To describe it concisely, we need a notational convention and a technical definition.

Convention 3.1. Let $T$ be a hypergeometric term whose kernel is $K$ and the corresponding shell is $S$. Then $T=S H$, where $H$ is a hypergeometric term whose shift quotient is $K$. Assume that $K$ is unequal to one. Moreover, write $K=u / v$, where $u, v$ are polynomials in $\mathbb{F}[y]$ with $\operatorname{gcd}(u, v)=1$.

Definition 3.2. A nonzero polynomial $p$ in $\mathbb{F}[y]$ is said to be strongly prime with $K$ if $\operatorname{gcd}\left(p, \sigma_{y}^{-i}(u)\right)=\operatorname{gcd}\left(p, \sigma_{y}^{i}(v)\right)=1$ for all $i \geq 0$.

The proof of Lemma 3 in [3] contains a reduction algorithm whose inputs and outputs are given below.

AbramovPetkovšekReduction: Given $K$ and $S$ as defined in Convention 3.1, compute a rational function $S_{1} \in \mathbb{F}(y)$ and polynomials $b, w \in \mathbb{F}[y]$ s.t. $b$ is shift-free and strongly prime with $K$, and the following equation holds:

$$
S=K \sigma_{y}\left(S_{1}\right)-S_{1}+\frac{w}{b \cdot \sigma_{y}^{-1}(u) \cdot v} .
$$

The algorithm contained in the proof of Lemma 3 in [3] is described as pseudo code on page 4 of the same paper, in which the last ten lines are to make the denominator of the rational function $V$ in its output minimal in some technical sense. We shall not execute these lines. Then the algorithm will compute two rational functions $U_{1}$ and $U_{2}$. They correspond to $S_{1}$ and $w /\left(b \sigma_{y}^{-1}(u) v\right)$ in (3), respectively.

We slightly modify the output of the Abramov-Petkovšek reduction. Note that $K$ is shift-reduced and $b$ is strongly prime with $K$. Thus, $b, \sigma_{y}^{-1}(u)$ and $v$ are pairwise coprime. By partial fraction decomposition, (3) can be rewritten as

$$
S=K \sigma_{y}\left(S_{1}\right)-S_{1}+\left(\frac{a}{b}+\frac{p_{1}}{\sigma_{y}^{-1}(u)}+\frac{p_{2}}{v}\right)
$$


where $a, p_{1}, p_{2} \in \mathbb{F}[y]$. Furthermore, we set $r=p_{1} / \sigma_{y}^{-1}(u)$. A direct calculation yields $r=K \sigma_{y}(-r)-(-r)+\sigma_{y}\left(p_{1}\right) / v$. Update $S_{1}$ to be $S_{1}-r$ and set $p$ to be $\sigma_{y}\left(p_{1}\right)+p_{2}$. Then

$$
S=K \sigma_{y}\left(S_{1}\right)-S_{1}+\frac{a}{b}+\frac{p}{v} .
$$

This modification leads to shell reduction specified below.

ShellReduction: Given $K$ and $S$ as defined in Convention 3.1, compute a rational function $S_{1} \in \mathbb{F}(y)$ and polynomials $a, b, p \in \mathbb{F}[y]$ s.t. $b$ is shift-free and strongly prime with $K$, and that (4) holds.

Shell reduction provides us with a necessary condition on summability.

Proposition 3.3. With Convention 3.1, assume that $a, b, p$ are polynomials in $\mathbb{F}[y]$ s.t. $b$ is shift-free and strongly prime with $K$. Assume further that (4) holds. If $T$ is summable, then $a / b$ belongs to $\mathbb{F}[y]$.

Proof. Recall that $T=S H$ by Convention 3.1 and it has a kernel $K$ and the corresponding shell $S$. It follows from (2) and (4) that $T \equiv(a / b+p / v) H \bmod \mathbb{U}_{H}$. Thus, $T$ is summable if and only if $(a / b+p / v) H$ is summable.

Set $H^{\prime}=(1 / v) H$, which has a kernel $K^{\prime}=u / \sigma_{y}(v)$. Note that $b$ is also strongly prime with $K^{\prime}$. We can apply Theorem 11 in [4] to $(a v / b+p) H^{\prime}$, which equals $(a / b+p / v) H$. Thus, $a / b$ is a polynomial because $b$ is coprime with $v$.

Example 3.4. Let $T=y^{2} y ! /(y+1)$. Then the term has a kernel $K=y+1$ and the shell $S=y^{2} /(y+1)$. Shell reduction yields $S \equiv-1 /(y+2)+y / v \bmod \mathbb{V}_{K}$ where $v=1$. By Proposition 3.3, $T$ is not summable.

Note that $a / b+p / v$ in (4) can be nonzero for a summable $T$.

Example 3.5. Let $T=y \cdot y$ ! whose kernel is $K=y+1$ and shell is $S=y$. Then $S \equiv y / v \bmod \mathbb{V}_{K}$, where $v=1$. But $T$ is summable as it is equal to $\Delta_{y}(y !)$.

The above example illustrates that neither shell reduction nor the Abramov-Petkovšek reduction can decide summability directly. One way to proceed is to find a polynomial solution of an auxiliary first-order linear difference equation [4]. We show how this can be avoided in the next section.

\section{MODIFICATIONS}

After the shell reduction described in (4), it remains to check the summability of $(a / b+p / v) H$. In the rational case, i.e. when the kernel $K$ is one, $a / b+p / v$ in (4) can be further reduced to $a / b$ with $\operatorname{deg}_{y}(a)<\operatorname{deg}_{y}(b)$, because all polynomials are rational summable. However, a hypergeometric term with a polynomial shell is not necessarily summable, for example, $y$ ! has a polynomial shell but it is not summable.

We define the notion of discrete residual forms for rational functions, and present a discrete variant of the polynomial reduction for hyperexponential functions given in [8]. This variant not only leads to a direct way to decide summability, but also reduces the number of terms of $p$ in (4).

\subsection{Discrete residual forms}

With Convention 3.1, we define an $\mathbb{F}$-linear map $\phi_{K}$ from $\mathbb{F}[y]$ to itself by sending $p$ to $u \sigma_{y}(p)-v p$ for all $p \in \mathbb{F}[y]$. We call $\phi_{K}$ the map for polynomial reduction w.r.t. $K$.
Lemma 4.1. Let

$\mathbb{W}_{K}=\operatorname{span}_{\mathbb{F}}\left\{y^{\ell} \mid \ell \in \mathbb{N}, \ell \neq \operatorname{deg}_{y}(p)\right.$ for all $\left.p \in \operatorname{im}\left(\phi_{K}\right)\right\}$.

Then $\mathbb{F}[y]=\operatorname{im}\left(\phi_{K}\right) \oplus \mathbb{W}_{K}$.

Proof. By the definition of $\mathbb{W}_{K}, \operatorname{im}\left(\phi_{K}\right) \cap \mathbb{W}_{K}=\{0\}$. The same definition also implies that, for every non-negative integer $m$, there exists a polynomial $f_{m} \in \operatorname{im}\left(\phi_{K}\right) \cup \mathbb{W}_{K}$ s.t. the degree of $f_{m}$ is equal to $m$. The set $\left\{f_{0}, f_{1}, f_{2}, \ldots\right\}$ forms an $\mathbb{F}$-basis of $\mathbb{F}[y]$. Thus $\mathbb{F}[y]=\operatorname{im}\left(\phi_{K}\right) \oplus \mathbb{W}_{K}$.

In view of the above lemma, we call $\mathbb{W}_{K}$ the standard complement of $\operatorname{im}\left(\phi_{K}\right)$. A polynomial $p \in \mathbb{F}$ can be uniquely decomposed as $p=p_{1}+p_{2}$ with $p_{1} \in \operatorname{im}\left(\phi_{K}\right)$ and $p_{2} \in \mathbb{W}_{K}$.

Lemma 4.2. With Convention 3.1, let $p$ be a polynomial in $\mathbb{F}[y]$. Then there exists $q \in \mathbb{W}_{K}$ s.t. $p / v \equiv q / v \bmod \mathbb{V}_{K}$.

Proof. Let $q$ be the projection of $p$ on $\mathbb{W}_{K}$. Then there exists $f$ in $\mathbb{F}[y]$ s.t. $p=\phi_{K}(f)+q$, that is, $p=u \sigma_{y}(f)-v f+q$. So $p / v=K \sigma_{y}(f)-f+q / v$, that is, $p / v \equiv q / v \bmod \mathbb{V}_{K}$.

Remark 4.3. Replacing $p$ in the above lemma by $v p$, we see that, for every polynomial $p \in \mathbb{F}[y]$, there exists $q \in \mathbb{W}_{K}$ s.t. $p \equiv q / v \bmod \mathbb{V}_{K}$.

By Lemma 4.2 and Remark 4.3, (4) implies that

$$
S \equiv \frac{a}{b}+\frac{q}{v} \bmod \mathbb{V}_{K}
$$

where $a, b, q \in \mathbb{F}[y], \operatorname{deg}_{y}(a)<\operatorname{deg}_{y}(b), b$ is shift-free and strongly prime with $K$, and $q \in \mathbb{W}_{K}$. The congruence (5) motivates us to translate the notion of (continuous) residual forms in [8] into the discrete setting.

Definition 4.4. With Convention 3.1, we further let $f$ be a rational function in $\mathbb{F}(y)$. Another rational function $r$ in $\mathbb{F}(y)$ is called a (discrete) residual form of $f$ w.r.t. $K$ if there exist $a, b, q$ in $\mathbb{F}[y]$ s.t.

$$
f \equiv r \bmod \mathbb{V}_{K} \quad \text { and } \quad r=\frac{a}{b}+\frac{q}{v},
$$

where $\operatorname{deg}_{y}(a)<\operatorname{deg}_{y}(b), b$ is shift-free and strongly prime with $K$, and $q$ belongs to $\mathbb{W}_{K}$. For brevity, we just say that $r$ is a residual form w.r.t. $K$ if $f$ is clear from the context.

Residual forms help us decide summability, as shown in the next proposition.

Proposition 4.5. With Convention 3.1, we further assume that $r$ is a nonzero residual form w.r.t. $K$. The hypergeometric term $r H$ is not summable.

Proof. Suppose that $r H$ is summable. Let $r=a / b+q / v$, where $\operatorname{deg}_{y}(a)<\operatorname{deg}_{y}(b), b$ is shift-free and strongly prime with $K$, and $q$ belongs to $\mathbb{W}_{K}$. By Proposition $3.3, a / b$ is a polynomial. Since $\operatorname{deg}_{y}(a)<\operatorname{deg}_{y}(b), a=0$. Thus, $(q / v) H$ is summable. It follows from (1) that there exists $w$ in $\mathbb{F}(y)$ s.t. $u \sigma_{y}(w)-v w=q$. Thus, $w \in \mathbb{F}[y]$ by Theorem 5.2.1 in $\left[19\right.$, page 76]. So $q$ belongs to im $\left(\phi_{K}\right)$. But $q$ also belongs to $\mathbb{W}_{K}$. By Lemma $4.1, q=0$, a contradiction.

With Convention 3.1, let $r$ be a residual form of the shell $S$. Then $S H \equiv r H \bmod \mathbb{U}_{H}$ by (2) and (5). By Proposition 4.5, $S H$ is summable if and only if $r=0$. Thus, determining the summability of a hypergeometric term $T$ amounts to computing a residual form of the corresponding shell w.r.t. a kernel of $T$, which is studied below. 


\subsection{Polynomial reduction}

To compute a residual form of a rational function, we project a polynomial on $\operatorname{im}\left(\phi_{K}\right)$ and on its standard complement $\mathbb{W}_{K}$, both defined in the previous subsection.

Let $\mathbb{B}_{K}=\left\{\phi_{K}\left(y^{i}\right) \mid i \in \mathbb{N}\right\}$. The $\mathbb{F}$-linear map $\phi_{K}$ is injective by Lemma 2.2. So $\mathbb{B}_{K}$ is an $\mathbb{F}$-basis of $\operatorname{im}\left(\phi_{K}\right)$, which allows us to construct an echelon basis. By an echelon basis, we mean an $\mathbb{F}$-basis in which distinct elements have distinct degrees. We can easily project a polynomial using an echelon basis and linear elimination.

To construct an echelon basis, we rewrite $\operatorname{im}\left(\phi_{K}\right)$ as

$$
\operatorname{im}\left(\phi_{K}\right)=\left\{u \Delta_{y}(p)-(v-u) p \mid p \in \mathbb{F}[y]\right\} .
$$

Set $\alpha_{1}=\operatorname{deg}_{y}(u), \alpha_{2}=\operatorname{deg}_{y}(v)$, and $\beta=\operatorname{deg}_{y}(v-u)$. Moreover, set $\tau_{K}=\mathrm{lc}_{y}(v-u) / \mathrm{lc}_{y}(u)$, which is nonzero due to Convention 3.1 and let $p$ be a nonzero polynomial in $\mathbb{F}[y]$.

We make the following case distinction.

Case 1. $\beta>\alpha_{1}$. Then $\beta=\alpha_{2}$, and

$$
\phi_{K}(p)=-\mathrm{lc}_{y}(v-u) \mathrm{lc}_{y}(p) y^{\alpha_{2}+\operatorname{deg}_{y}(p)}+\text { lower terms. }
$$

So $\mathbb{B}_{K}$ is an echelon basis of $\operatorname{im}\left(\phi_{K}\right)$, in which $\operatorname{deg}_{y}\left(\phi_{K}\left(y^{i}\right)\right)$ is equal to $\alpha_{2}+i$ for all $i \in \mathbb{N}$. Accordingly, $\mathbb{W}_{K}$ has an echelon basis $\left\{1, y, \ldots, y^{\alpha_{2}-1}\right\}$ and $\operatorname{dim}\left(\mathbb{W}_{K}\right)=\alpha_{2}$.

Case 2. $\beta=\alpha_{1}$. Then

$$
\phi_{K}(p)=-\mathrm{lc}_{y}(v-u) \mathrm{lc}_{y}(p) y^{\alpha_{1}+\operatorname{deg}_{y}(p)}+\text { lower terms. }
$$

So $\mathbb{B}_{K}$ is an echelon basis of $\operatorname{im}\left(\phi_{K}\right)$, in which $\operatorname{deg}_{y}\left(\phi_{K}\left(y^{i}\right)\right)$ is equal to $\alpha_{1}+i$ for all $i \in \mathbb{N}$. Accordingly, $\mathbb{W}_{K}$ has an echelon basis $\left\{1, y, \ldots, y^{\alpha_{1}-1}\right\}$ and $\operatorname{dim}\left(\mathbb{W}_{K}\right)=\alpha_{1}$.

Case 3. $\beta<\alpha_{1}-1$. If $\operatorname{deg}_{y}(p)=0$, then $\phi_{K}(p)=(u-v) p$. Otherwise, we have

$$
\phi_{K}(p)=\operatorname{deg}_{y}(p) \operatorname{lc}_{y}(u) \operatorname{lc}_{y}(p) y^{\alpha_{1}+\operatorname{deg}_{y}(p)-1}+\text { lower terms. }
$$

It follows that $\mathbb{B}_{K}$ is an echelon basis of $\operatorname{im}\left(\phi_{K}\right)$, in which $\operatorname{deg}_{y}\left(\phi_{K}(1)\right)=\beta$ and

$$
\operatorname{deg}_{y}\left(\phi_{K}\left(y^{i}\right)\right)=\alpha_{1}+i-1 \quad \text { for all } i \geq 1 .
$$

So $\mathbb{W}_{K}$ has an echelon basis $\left\{1, \ldots, y^{\beta-1}, y^{\beta+1}, \ldots, y^{\alpha_{1}-1}\right\}$, and $\operatorname{dim}\left(\mathbb{W}_{K}\right)=\alpha_{1}-1$.

Case 4. $\beta=\alpha_{1}-1$ and $\tau_{K}$ is not a positive integer. Then

$$
\begin{aligned}
\phi_{K}(p)= & \left(\operatorname{deg}_{y}(p) \operatorname{lc}_{y}(u)-\operatorname{lc}_{y}(v-u)\right) \operatorname{lc}_{y}(p) y^{\alpha_{1}+\operatorname{deg}_{y}(p)-1} \\
& + \text { lower terms. }
\end{aligned}
$$

So $\mathbb{B}_{K}$ is an echelon basis of $\operatorname{im}\left(\phi_{K}\right)$, in which, for all $i \in \mathbb{N}$, $\operatorname{deg}_{y}\left(\phi_{K}\left(y^{i}\right)\right)=\alpha_{1}+i-1$. Accordingly, $\mathbb{W}_{K}$ is spanned by an echelon basis $\left\{1, y, \ldots, y^{\alpha_{1}-2}\right\}$, and has dimension $\alpha_{1}-1$. Case 5. $\beta=\alpha_{1}-1$ and $\tau_{K}$ is a positive integer. It follows from (6) that for $i \neq \tau_{K}, \operatorname{deg}_{y}\left(\phi_{K}\left(y^{i}\right)\right)=\alpha_{1}+i-1$. Moreover, for every polynomial $p$ of degree $\tau_{K}, \phi_{K}(p)$ is of degree less than $\alpha_{1}+\tau_{K}-1$. So any echelon basis of $\operatorname{im}\left(\phi_{K}\right)$ does not contain a polynomial of degree $\alpha_{1}+\tau_{K}-1$. Set

$$
\mathbb{B}_{K}^{\prime}=\left\{\phi_{K}\left(y^{i}\right) \mid i \in \mathbb{N}, i \neq \tau_{K}\right\} .
$$

Reducing $\phi_{K}\left(y^{\tau_{K}}\right)$ by the polynomials in $\mathbb{B}_{K}^{\prime}$, we obtain a polynomial $p^{\prime}$ with $\operatorname{deg}_{y}\left(p^{\prime}\right)<\alpha_{1}-1$. Since $\mathbb{B}_{K}$ is an $\mathbb{F}$ basis and $\mathbb{B}_{K}^{\prime} \subset \mathbb{B}_{K}, p^{\prime} \neq 0$. So $\mathbb{B}_{K}^{\prime} \cup\left\{p^{\prime}\right\}$ is an echelon basis of $\operatorname{im}\left(\phi_{K}\right)$. Consequently, $\mathbb{W}_{K}$ is spanned by an echelon ba$\operatorname{sis}\left\{1, y, \ldots, y^{\operatorname{deg}_{y}\left(p^{\prime}\right)-1}, y^{\operatorname{deg}_{y}\left(p^{\prime}\right)+1}, \ldots, y^{\alpha_{1}-2}, y^{\alpha_{1}+\tau_{K}-1}\right\}$. The dimension of $\mathbb{W}_{K}$ is equal to $\alpha_{1}-1$.
Example 4.6. Let $K=\left(y^{4}+1\right) /(y+1)^{4}$, which is shiftreduced. Then $\tau_{K}=4$. According to Case $5, \mathrm{im}\left(\phi_{K}\right)$ has an echelon basis

$$
\left\{\phi_{K}(p)\right\} \cup\left\{\phi_{K}\left(y^{m}\right) \mid m \in \mathbb{N}, m \neq 4\right\},
$$

where $p=y^{4}+y / 3+1 / 2, \phi_{K}(p)=(5 / 3) y^{2}+2 y+4 / 3$, and $\phi_{K}\left(y^{m}\right)=(m-4) y^{m+3}+$ lower terms. Therefore, $\mathbb{W}_{K}$ has a basis $\left\{1, y, y^{7}\right\}$.

From the above case distinction and example, one observes that, although the degree of a polynomial in the standard complement depends on $\tau_{K}$, which may be arbitrarily high, the number of its terms depends merely on the degrees of $u$ and $v$. We record this observation in the next proposition.

Proposition 4.7. With the Convention 3.1, we further let $\alpha_{1}=\operatorname{deg}_{y}(u), \quad \alpha_{2}=\operatorname{deg}_{y}(v), \quad$ and $\beta=\operatorname{deg}_{y}(v-u)$.

Then there exists $\mathcal{P} \subset\left\{y^{i} \mid i \in \mathbb{N}\right\}$ with

$$
|\mathcal{P}| \leq \max \left\{\alpha_{1}, \alpha_{2}\right\}-\llbracket \beta \leq \alpha_{1}-1 \rrbracket
$$

s.t. every polynomial in $\mathbb{F}[y]$ can be reduced modulo $\operatorname{im}\left(\phi_{K}\right)$ to an $\mathbb{F}$-linear combination of the elements in $\mathcal{P}$. Note that here $\llbracket \beta \leq \alpha_{1}-1 \rrbracket$ equals 1 if $\beta \leq \alpha_{1}-1$, otherwise it is 0 .

Proof. By the above case distinction, $\operatorname{dim}\left(\mathbb{W}_{K}\right)$ is no more than $\max \left\{\alpha_{1}, \alpha_{2}\right\}-\llbracket \beta \leq \alpha_{1}-1 \rrbracket$. The lemma follows.

The above case distinction enables one to find an infinite sequence $p_{0}, p_{1}, \ldots$ in $\mathbb{F}[y]$ s.t.

$$
\mathbb{E}_{K}=\left\{\phi_{K}\left(p_{i}\right) \mid i \in \mathbb{N}\right\} \text { with } \operatorname{deg}_{y} \phi_{K}\left(p_{i}\right)<\operatorname{deg}_{y} \phi_{K}\left(p_{i+1}\right),
$$

is an echelon basis of $\mathrm{im}\left(\phi_{K}\right)$. This basis allows us to project a polynomial on $\operatorname{im}\left(\phi_{K}\right)$ and $\mathbb{W}_{K}$, respectively. In the first four cases, the $p_{i}$ 's can be chosen as powers of $y$. But in the last case, one of the $p_{i}$ 's is not necessarily a monomial as shown in Example 4.6.

PolynomialReduction: Given $p \in \mathbb{F}[y]$, compute $f \in \mathbb{F}[y]$ and $q \in \mathbb{W}_{K}$ s.t. $p=\phi_{K}(f)+q$.

1. If $p=0$, then set $f=0$ and $q=0$; return.

2. Set $d=\operatorname{deg}_{y}(p)$. Find the subset $\mathbb{P}=\left\{p_{i_{1}}, \ldots, p_{i_{s}}\right\}$ consisting of the preimages of all polynomials in the echelon basis $\mathbb{E}_{K}$ whose degrees are at most $d$.

3. For $k=s, s-1, \ldots, 1$, perform linear elimination to find $c_{s}, c_{s-1}, \ldots, c_{1} \in \mathbb{F}$ s.t. $p-\sum_{k=1}^{s} c_{k} \phi_{K}\left(p_{i_{k}}\right) \in \mathbb{W}_{K}$.

4. Set $f=\sum_{k=1}^{s} c_{k} p_{i_{k}}$ and $q=p-\phi_{K}(f)$; and return.

We now present a modified version of the Abramov-Petkovšek reduction, which determines summability without solving any auxiliary difference equations explicitly.

ModifiedAbramovPetkovšekReduction: Given an irrational hypergeometric term $T$ over $\mathbb{F}(y)$, compute a hypergeometric term $H$ with a kernel $K$, and two rational functions $f, r \in \mathbb{F}(y)$ s.t. $r$ is a residual form w.r.t. $K$, and

$$
T=\Delta_{y}(f H)+r H .
$$

1. Find a kernel $K$ and the corresponding shell $S$ of $T$; 
2. Apply shell reduction to $S$ w.r.t. $K$ to find $b, s, t \in \mathbb{F}[y]$ and $g \in \mathbb{F}(y)$ s.t. $b$ is shift-free and strongly prime with $K$; and

$$
T=\Delta_{y}(g H)+\left(\frac{s}{b}+\frac{t}{v}\right) H
$$

where $\sigma_{y}(H) / H=K$ and $v$ is the denominator of $K$.

3. Set $p$ and $a$ to be the quotient and remainder of $s$ and $b$, respectively.

4. Apply polynomial reduction to $v p+t$ to find $h \in \mathbb{F}[y]$ and $q \in \mathbb{W}_{K}$ s.t. $v p+t=\phi_{K}(h)+q$.

5. Set $f:=g+h$ and $r:=a / b+q / v$ and return $H, f$ and $r$.

Theorem 4.8. With Convention 3.1, the modified version of the Abramov-Petkovšek reduction computes a rational function $f$ in $\mathbb{F}(y)$ and a residual form $r$ w.r.t. $K$ s.t. (7) holds. Moreover, $T$ is summable if and only if $r=0$.

Proof. Recall that $T=S H$, where $H$ has a kernel $K$ and $S$ is a rational function. Applying shell reduction to $S$ w.r.t. $K$ yields (8), which can be rewritten as

$$
T=\Delta_{y}(g H)+\left(\frac{a}{b}+\frac{v p+t}{v}\right) H
$$

where $a$ and $p$ are given in step 3 of the modified AbramovPetkovšek reduction. The polynomial reduction in step 4 yields that $v p+t=u \sigma_{y}(h)-v h+q$. Substituting this into (8), we see that

$$
\begin{aligned}
T & =\Delta_{y}(g H)+\left(K \sigma_{y}(h)-h\right) H+\left(\frac{a}{b}+\frac{q}{v}\right) H \\
& =\Delta_{y}((g+h) H)+r H,
\end{aligned}
$$

where $r=a / b+q / v$. Thus, (7) holds. By Proposition 4.5, T is summable if and only $r$ is equal to zero.

Example 4.9. Let $T$ be the same hypergeometric term as in Example 3.4. Then $K=y+1$ and $S=y^{2} /(y+1)$. Set $H=y !$. By the shell reduction in Example 3.4,

$$
T=\Delta_{y}\left(\frac{-1}{y+1} H\right)+\left(\frac{-1}{y+2}+\frac{y}{v}\right) H,
$$

where $v=1$. Applying the polynomial reduction to $(y / v) H$ yields $(y / v) H=\Delta_{y}(1 \cdot H)$. Combining the above steps, we decompose $T$ as $T=\Delta_{y}(y /(y+1) H)-(1 /(y+2)) H$. So the input term $T$ is not summable.

Example 4.10. Let $T$ be the same hypergeometric term as in Example 3.5. Then $K=y+1$ and $S=y$. Set $H=y$ !. By the shell reduction in Example 3.5, $T=y H$. The polynomial reduction yields $y H=\Delta_{y}(y !)$, hence $T=\Delta_{y}(y !)$.

Remark 4.11. With the notation given in the step 5 of the modified version, we can rewrite $r H$ as $\left(s_{1} / s_{2}\right) G$, where $s_{1}=a v+b q, s_{2}=b$, and $G=H / v$. It follows from the case distinction at the beginning of this section that the degree of $s_{1}$ is bounded by $\lambda$ given in [3, Theorem 8]. The polynomial $s_{2}$ is equal to $b$ in (3) whose degree is minimal by [3, Theorem 3]. Moreover, $\sigma_{y}(G) / G$ is shift-reduced, because $\sigma_{y}(H) / H$ is. These are exactly the same required properties of the output of the original version [3].

\section{SUM OF TWO RESIDUAL FORMS}

To compute telescopers for bivariate hypergeometric terms by the modified Abramov-Petkovšek reduction, we are confronted with the difficulty that the sum of two residual forms is not necessarily a residual form. This is because the least common multiple of two shift-free polynomials is not necessarily shift-free.

The goal of this section is to show that the sum of two residual forms is congruent to a residual form modulo $\mathbb{V}_{K}$.

Example 5.1. Let $K=1 / y, r=1 /(2 y+1)$ and $s=1 /(2 y+3)$. Then both $r$ and $s$ are residual forms w.r.t. $K$, but their sum is not, because the denominator $(2 y+1)(2 y+3)$ is not shiftfree. However, we can still find an equivalent residual form. For example, we have $r+s \equiv-1 /(2(2 y+1))+1 / 2 y \bmod \mathbb{V}_{K}$. Note that the residual form is not unique. Another possible choice is $r+s \equiv 1 /(3(2 y+3))+1 / 3 y \bmod \mathbb{V}_{K}$.

Let $f$ and $g$ be two nonzero polynomials in $\mathbb{F}[y]$. We say that $f$ and $g$ are shift-coprime if $\operatorname{gcd}\left(f, \sigma_{y}^{\ell}(g)\right)=1$ for all nonzero integer $\ell$. Assume that both $f$ and $g$ are shift-free. By polynomial factorization and dispersion computation, one can uniquely decompose

$$
g=\tilde{g} \sigma_{y}^{\ell_{1}}\left(p_{1}^{m_{1}}\right) \cdots \sigma_{y}^{\ell_{k}}\left(p_{k}^{m_{k}}\right),
$$

where $\tilde{g}$ is shift-coprime with $f, p_{1}, \ldots, p_{k}$ are distinct, monic and irreducible factors of $f, \ell_{1}, \ldots, \ell_{k}$ are nonzero integers, $m_{1}, \ldots, m_{k}$ are multiplicities of $\sigma_{y}^{\ell_{1}}\left(p_{1}\right), \ldots, \sigma_{y}^{\ell_{k}}\left(p_{k}\right)$ in $g$, respectively. We refer to (9) as the shift-coprime decomposition of $g$ w.r.t. $f$.

Remark 5.2. The factors $\tilde{g}, \sigma_{y}^{\ell_{1}}\left(p_{1}^{m_{1}}\right), \ldots, \sigma_{y}^{\ell_{k}}\left(p_{k}^{m_{k}}\right)$ in (9) are pairwise coprime, since $f$ and $g$ are shift-free.

To construct a residual form congruent to the sum of two given residual ones, we need three technical lemmas. The first one corresponds to the kernel reduction in [8].

Lemma 5.3. With Convention 3.1, assume that $p_{1}, p_{2}$ are in $\mathbb{F}[y]$ and $m$ in $\mathbb{N}$. Then there exist $q_{1}, q_{2}$ in $\mathbb{W}_{K}$ s.t.

$\frac{p_{1}}{\prod_{i=0}^{m} \sigma_{y}^{i}(v)} \equiv \frac{q_{1}}{v} \bmod \mathbb{V}_{K}$ and $\frac{p_{2}}{\prod_{j=1}^{m} \sigma_{y}^{-j}(u)} \equiv \frac{q_{2}}{v} \bmod \mathbb{V}_{K}$.

Proof. To prove the first congruence, let $w_{m}=\prod_{i=0}^{m} \sigma_{y}^{i}(v)$.

We proceed by induction on $m$. If $m=0$, then the conclusion holds by Lemma 4.2. Assume that the lemma holds for $m-1$. Consider the equality

$$
\frac{p_{1}}{w_{m}}=K \sigma_{y}\left(\frac{s}{w_{m-1}}\right)-\frac{s}{w_{m-1}}+\frac{t}{w_{m-1}},
$$

where $s, t \in \mathbb{F}[y]$ are to be determined. This equality holds if and only if $\sigma_{y}(s) u+(t-s) \sigma_{y}^{m}(v)=p_{1}$. Since $u$ and $\sigma_{y}^{m}(v)$ are coprime, such $s$ and $t$ can be computed by the extended Euclidean algorithm. Thus, $p_{1} / w_{m} \equiv t / w_{m-1} \bmod \mathbb{V}_{K}$. Consequently, $p_{1} / w_{m}$ has a required residual form by the induction hypothesis.

To prove the second congruence, we use the identity

$$
\frac{p_{2}}{\sigma_{y}^{-1}(u)}=K \sigma_{y}\left(-\frac{p_{2}}{\sigma_{y}^{-1}(u)}\right)-\left(-\frac{p_{2}}{\sigma_{y}^{-1}(u)}\right)+\frac{\sigma_{y}\left(p_{2}\right)}{v},
$$

which implies that $p_{2} / \sigma_{y}^{-1}(u) \equiv \sigma_{y}\left(p_{2}\right) / v \bmod \mathbb{V}_{K}$. By Lemma 4.2 , there exists $q_{2} \in \mathbb{W}_{K}$ s.t. $q_{2} / v$ is a residual form of $p_{2} / \sigma_{y}^{-1}(u)$ w.r.t. $K$. Assume that the congruence holds for $m-1$. The induction can be completed as in the proof for $p_{1} / w_{m}$. 
The next lemma provides us with flexibility to rewrite a rational function modulo $\mathbb{V}_{K}$.

Lemma 5.4. Let $K \in \mathbb{F}(y)$ be nonzero and shift-reduced. Then, for every $f \in \mathbb{F}(y)$ and every $\ell \in \mathbb{Z}^{+}$,

$$
f \equiv \sigma_{y}^{\ell}(f) \prod_{i=0}^{\ell-1} \sigma_{y}^{i}(K) \equiv \sigma_{y}^{-\ell}(f) \prod_{i=1}^{\ell} \sigma_{y}^{-i}\left(\frac{1}{K}\right) \bmod \mathbb{V}_{K} .
$$

Proof. Let us show the first congruence by induction on $\ell$. For $\ell=1$, the identity $f=K \sigma_{y}(-f)-(-f)+\sigma_{y}(f) K$ implies that $f$ is congruent to $\sigma_{y}(f) K$ modulo $\mathbb{V}_{K}$. Assume that it holds for $\ell-1$. Set $w_{\ell}=\prod_{i=0}^{\ell-1} \sigma_{y}^{i}(K)$. Then $f$ is congruent to $\sigma_{y}^{\ell-1}(f) w_{\ell-1}$ modulo $\mathbb{V}_{K}$ by the induction hypothesis. Moreover, $\sigma_{y}^{\ell-1}(f) w_{\ell-1}$ is congruent to $\sigma_{y}^{\ell}(f) w_{\ell}$ by the induction base, in which $f$ is replaced with $\sigma_{y}^{\ell-1}(f) w_{\ell-1}$. Hence, $f$ is congruent to $\sigma_{y}^{\ell}(f) w_{\ell}$ modulo $\mathbb{V}_{K}$.

The second congruence can be shown similarly. For $\ell=1$, the identity $f=K \sigma_{y}(r)-r+r$ with $r=\sigma_{y}^{-1}(f) \sigma_{y}^{-1}(1 / K)$ implies that $f$ is congruent to $r$ modulo $\mathbb{V}_{K}$. We can then proceed as in the proof of the first congruence.

Lemma 5.5. With Convention 3.1, let $a, b \in \mathbb{F}[y]$ with $b \neq 0$. Assume that $b$ is shift-free and strongly prime with $K$. Assume further that $\sigma_{y}^{\ell}(b)$ is strongly prime with $K$ for some integer $\ell$, then $a / b$ has a residual form $c / \sigma_{y}^{\ell}(b)+q / v$ w.r.t. $K$, where $c \in \mathbb{F}[y]$ with $\operatorname{deg}_{y}(c)<\operatorname{deg}_{y}(b)$ and $q \in \mathbb{W}_{K}$.

Proof. First, consider the case in which $\ell \geq 0$. If $\ell=0$, then there exist $c, p \in \mathbb{F}$ with $\operatorname{deg}_{y}(c)<\operatorname{deg}_{y}(b)$ s.t. $a / b=c / b+p$. The lemma follows from Remark 4.3.

Assume that $\ell>0$. By the first congruence of Lemma 5.4,

$$
\frac{a}{b} \equiv \sigma_{y}^{\ell}\left(\frac{a}{b}\right)\left(\prod_{i=0}^{\ell-1} \sigma_{y}^{i}(K)\right)=\frac{\sigma_{y}^{\ell}(a)}{\sigma_{y}^{\ell}(b)} \frac{\prod_{i=0}^{\ell-1} \sigma_{y}^{i}(u)}{\prod_{i=0}^{\ell-1} \sigma_{y}^{i}(v)} \bmod \mathbb{V}_{K}
$$

Note that $\sigma_{y}^{\ell}(b)$ is strongly prime with $v$ by assumption. Then it is coprime with the product $v \sigma_{y}(v) \cdots \sigma_{y}^{\ell-1}(v)$. By partial fraction decomposition, we get

$$
\frac{a}{b} \equiv \frac{\tilde{a}}{\sigma_{y}^{\ell}(b)}+\frac{\tilde{q}}{\prod_{i=0}^{\ell-1} \sigma_{y}^{i}(v)} \bmod \mathbb{V}_{K} .
$$

By the first congruence of Lemma 5.3, the second summand in the right-hand side of the above congruence can be replaced by a residual form whose denominator is equal to $v$. The first assertion holds.

The case in which $\ell<0$ can be handled in the same way, in which the second congruences of Lemmas 5.4 and 5.3 will be used instead of the first ones in these lemmas.

We are ready to present the main result of this section.

Theorem 5.6. With Convection 3.1, let $r$ and $s$ be two residual forms w.r.t. $K$. Then there exists a residual form $t$ congruent to $s$ modulo $\mathbb{V}_{K}$ s.t., for all $\lambda, \mu \in \mathbb{F}, \lambda r+\mu t$ is a residual form w.r.t. $K$ congruent to $\lambda r+\mu s$ modulo $\mathbb{V}_{K}$.

Proof. Let $r=a / f+p / v$ and $s=b / g+q / v$, where $a, f, b, g \in$ $\mathbb{F}[y], \operatorname{deg}_{y}(a)<\operatorname{deg}(f), \operatorname{deg}_{y}(b)<\operatorname{deg}_{y}(g), p, q \in \mathbb{W}_{K}$, and $f, g$ are shift-free and strongly prime with $K$.

Assume that (9) is the shift-coprime decomposition of $g$ w.r.t. $f$. Set $P_{i}=\sigma_{y}^{\ell_{i}}\left(p_{i}\right)$ for $i=1, \ldots, k$. By Remark 5.2 and partial fraction decomposition, we have

$$
\frac{b}{g}=\frac{b_{0}}{\tilde{g}}+\sum_{i=1}^{k} \frac{b_{i}}{P_{i}^{m_{i}}}
$$

where $b_{0}, b_{1}, \ldots, b_{k} \in \mathbb{F}[y]$. Note that $p_{i}=\sigma_{y}^{-\ell_{i}}\left(P_{i}\right)$, which is a factor of $f$. Thus it is strongly prime with $K$. So we can apply Lemma 5.5 to each fraction $b_{i} / P_{i}^{m_{i}}$ in (10) to get

$$
\frac{b}{g} \equiv \frac{b_{0}}{\tilde{g}}+\sum_{i=1}^{k} \frac{b_{i}^{\prime}}{p_{i}^{m_{i}}}+\frac{q^{\prime}}{v} \bmod \mathbb{V}_{K}
$$

where $b_{1}^{\prime}, \ldots, b_{k}^{\prime} \in \mathbb{F}[y]$ and $q^{\prime} \in \mathbb{W}_{K}$.

Set $h=\tilde{g} \prod_{i=1}^{k} p_{i}^{m_{i}}$. Then $h$ is shift-free and strongly prime with $K$ as both $f$ and $g$ are. Since $f$ is shift-free, all its factors are shift-coprime with $f$, so are the $p_{i}$ 's, and so is $h$. Let $t$ be the sum of $q / v$ and the rational function in the right-hand side of (11). Then there exist $b^{*}$ in $\mathbb{F}[y]$ with $\operatorname{deg}_{y}\left(b^{*}\right)<\operatorname{deg}_{y}(h)$ and $q^{*}$ in $\mathbb{W}_{K}$ s.t. $t=b^{*} / h+q^{*} / v$. Since $f$ and $h$ are shift-coprime, their least common multiple is shift-free. Therefore, $\lambda r+\mu t$ is a residual form w.r.t. $K$, and $\lambda r+\mu t$ is congruent to $\lambda r+\mu s \bmod \mathbb{V}_{K}$.

\section{TELESCOPING VIA REDUCTIONS}

Let $\mathcal{C}$ be a field of characteristic zero, and $\mathcal{C}(x, y)$ be the field of rational functions in $x$ and $y$ over $\mathcal{C}$. Let $\sigma_{x}, \sigma_{y}$ be the shift operators w.r.t. $x$ and $y$, respectively, defined by,

$$
\sigma_{x}(f(x, y))=f(x+1, y) \text { and } \sigma_{y}(f(x, y))=f(x, y+1),
$$

for any $f \in \mathcal{C}(x, y)$. Then the pair $\left(\mathcal{C}(x, y),\left\{\sigma_{x}, \sigma_{y}\right\}\right)$ forms a partial difference field.

Definition 6.1. Let $\mathbb{D}$ be a partial difference ring extension of $\mathcal{C}(x, y)$. A nonzero element $T \in \mathbb{D}$ is called a hypergeometric term over $\mathcal{C}(x, y)$ if there exist $f, g \in \mathcal{C}(x, y)$ s.t. $\sigma_{x}(T)=f T$ and $\sigma_{y}(T)=g T$. We call $f, g$ the shift quotients of $T$ w.r.t. $x$ and $y$, respectively.

An irreducible polynomial $p \in \mathcal{C}[x, y]$ is said to be integerlinear over $\mathcal{C}$ if there exist $f \in \mathcal{C}[z], m, n \in \mathbb{Z}$ with $n \geq 0$ and $\operatorname{gcd}(m, n)=1$, s.t. $p=f(m x+n y)$. A polynomial in $\mathcal{C}[x, y]$ is said to be integer-linear over $\mathcal{C}$ if all of its irreducible factors are integer-linear. A rational function in $\mathcal{C}(x, y)$ is said to be integer-linear over $\mathcal{C}$ if its denominator and numerator are integer-linear.

Let $\mathbb{F}$ be the field $\mathcal{C}(x)$, and $\mathbb{F}\left\langle S_{x}\right\rangle$ be the ring of linear recurrence operators in $x$, in which the commutation rule is that $S_{x} r=\sigma_{x}(r) S_{x}$ for all $r \in \mathbb{F}$. The application of an operator $L=\sum_{i=0}^{\rho} \ell_{i} S_{x}^{i}$ to a hypergeometric term $T$ is defined as $L(T)=\sum_{i=0}^{\rho} \ell_{i} \sigma_{x}^{i}(T)$.

Definition 6.2. Let $T$ be a hypergeometric term over $\mathbb{F}(y)$. A nonzero operator $L \in \mathbb{F}\left\langle S_{x}\right\rangle$ is called a telescoper for $T$ if there exists a hypergeometric term $G$ s.t. $L(T)=\Delta_{y}(G)$. We call $G$ the certificate of $L$.

For hypergeometric terms, telescopers do not always exist. Abramov presented a criterion for determining the existence of telescopers in [2, Theorem 10]. Let $K=u / v$ be a kernel of $\sigma_{y}(T) / T$ and $S$ the corresponding shell. Applying the modified Abramov-Petkovšek reduction w.r.t. $y$ to $T$ yields $T=\Delta_{y}(u H)+r H$, where $u \in \mathbb{F}(y), H=T / S$, and $r=a / b+q / v$ is the residual form of $S$ w.r.t. $K$. By Abramov's criterion, $T$ has a telescoper if and only if $b$ is 
integer-linear over $\mathcal{C}$. When telescopers exist, Zeilberger's algorithm [21] constructs a telescoper for $T$ by iteratively using the Gosper algorithm to detect the summability of $L(T)$ for an ansatz $L=\sum_{i=0}^{\rho} \ell_{i} S_{x}^{i} \in \mathbb{F}\left\langle S_{x}\right\rangle$.

Following the creative telescoping algorithms based on Hermite reductions $[7,10,9,8]$ in the continuous case, we use the modified Abramov-Petkovšek reduction to develop a telescoping algorithm, which is outlined below.

ReductionCT: Given a hypergeometric term $T$ with shift quotients $f=\sigma_{x}(T) / T$ and $g=\sigma_{y}(T) / T$ in $\mathbb{F}(y)$, compute a telescoper of minimal order for $T$ and its certificate if telescopers exist.

1. Find a kernel $K$ and shell $S$ of $T$ w.r.t. $y$ s.t. $T=S H$ with $K=\sigma_{y}(H) / H$.

2. Apply the modified Abramov-Petkovšek reduction to $T$ to get

$$
T=\Delta_{y}\left(u_{0} H\right)+r_{0} H
$$

If $r_{0}=0$, then return $\left(1, u_{0} H\right)$.

3 . If the denominator of $r_{0}$ is not integer-linear, return "No telescoper exists!".

4. Set $N:=\sigma_{x}(H) / H$ and $R:=\ell_{0} r_{0}$, where $\ell_{0}$ is an indeterminate.

For $i=1,2, \ldots$, do

4.1. View $\sigma_{x}\left(r_{i-1}\right) N H$ as a hypergeometric term with kernel $K$ and shell $\sigma_{x}\left(r_{i-1}\right) N$. Using shell reduction w.r.t. $K$ and polynomial reduction w.r.t. $K$, find $u_{i}^{\prime} \in \mathbb{F}$ and a residual form $\tilde{r}_{i}$ w.r.t. $K$ s.t. $\sigma_{x}\left(r_{i-1}\right) N H=\Delta_{y}\left(u_{i}^{\prime} H\right)+\tilde{r}_{i} H$.

4.2. Set $\tilde{u}_{i}=\sigma_{x}\left(u_{i-1}\right) N+u_{i}^{\prime}$, so that

$$
\sigma_{x}^{i}(T)=\Delta_{y}\left(\tilde{u}_{i} H\right)+\tilde{r}_{i} H .
$$

4.3. Follow the proof of Theorem 5.6 to compute $u_{i}$ and $r_{i}$ in $\mathbb{F}(y)$ s.t. $r_{i} \equiv \tilde{r}_{i} \bmod \mathbb{V}_{K}$,

$$
\sigma_{x}^{i}(T)=\Delta_{y}\left(u_{i} H\right)+r_{i} H,
$$

and that $R+\ell_{i} r_{i}$ is a residual form w.r.t. $K$, where $\ell_{i}$ is an indeterminate.

4.4. Update $R$ to be $R+\ell_{i} r_{i}$.

4.5. Find $\ell_{j} \in \mathbb{F}$ s.t. $R=0$ by solving a linear system in $\ell_{0}, \ldots, \ell_{i}$ over $\mathbb{F}$. If there is a nontrivial solution, return $\left(\sum_{j=0}^{i} \ell_{j} S_{x}^{j}, \sum_{j=0}^{i} \ell_{j} u_{j} H\right)$.

Theorem 6.3. Let $T$ be a hypergeometric term over $\mathbb{F}(y)$. If $T$ has a telescoper, then the algorithm ReductionCT terminates and returns a telescoper of minimal order for $T$.

Proof. By Theorem 4.8, $r_{0}=0$ implies that 1 is a telescoper for $T$ of minimal order.

Let $r_{0}$ obtained from step 2 be of the form $a_{0} / b_{0}+q_{0} / v$, where $a_{0}, b_{0}, v \in \mathbb{F}[y], \operatorname{deg}_{y}\left(a_{0}\right)<\operatorname{deg}_{y}\left(b_{0}\right), b_{0}$ is strongly prime with $K, q_{0} \in \mathbb{W}_{K}$, and $v$ is the denominator of $K$. By Ore-Sato's theorem $[18,20]$ on hypergeometric terms, $K$ is integer-linear and so is $v$. It follows that $b_{0}$ is integerlinear if and only if $b_{0} v$ is. By Abramov's criterion, $T$ has a telescoper if and only if the denominator of $r_{0}$ is integerlinear. Thus, steps 2 and 3 are correct.

It follows from (12) and $\sigma_{x}\left(r_{0} H\right)=\sigma_{x}\left(r_{0}\right) N H$ that (13) holds for $i=1$. By Theorem 5.6, there exists a residual form $r_{1}$ w.r.t. $K$ with $r_{1} \equiv \tilde{r}_{1} \bmod \mathbb{V}_{K}$ s.t. $R+\ell_{1} r_{1}$ is again a residual form for all $\ell_{0}, \ell_{1} \in \mathbb{F}$. Indeed, the proofs of the lemmas and Theorem 5.6 enable us to obtain not only $r_{1}$ but also a rational function $g_{1}$ s.t. $\tilde{r}_{1}=K \sigma_{y}\left(g_{1}\right)-g_{1}+r_{1}$. Setting $u_{1}=\tilde{u}_{1}+g_{1}$, we see that (14) holds for $i=1$. By a direct induction on $i,(14)$ holds in the loop of step 4 .

Assume that $L=\sum_{i=0}^{\rho} c_{i} S_{x}^{i}$ is a telescoper of minimal order for $T$ with $c_{i} \in \mathbb{F}$ and $c_{\rho} \neq 0$. Then $L(T)$ is summable. By Theorem 4.8, $\sum_{i=0}^{\rho} c_{i} r_{i}$ is equal to zero. Thus, the linear homogeneous system (over $\mathbb{F}$ ) obtained by equating $\sum_{i=0}^{\rho} \ell_{i} r_{i}$ to zero has a nontrivial solution, which yields a telescoper of minimal order.

Remark 6.4. The algorithm ReductionCT separates the computation of minimal telescopers from that of certificates. In applications where the certificates are irrelevant, we can drop step 4.2 and in step 4.3 compute $u_{i}$ and $r_{i}$ with $r_{i} \equiv$ $\tilde{r}_{i} \bmod \mathbb{V}_{K}$ and $\sigma_{x}^{i}\left(r_{i-1}\right) N H=\Delta_{y}\left(u_{i} H\right)+r_{i} H$ and that $R+$ $\ell_{i} r_{i}$ is a residual form w.r.t. $K$, where $\ell_{i}$ is an indeterminate. The rational function $u_{i}$ can be discarded, and we do not need to calculate $\sum_{j=0}^{i} \ell_{j} u_{j} H$ in the end.

Remark 6.5. Instead of applying the modified AbramovPetkovšek reduction to $\sigma_{x}\left(r_{i-1}\right) N H$ in step 4.1, it is also possible to apply the reduction to $\sigma_{x}^{i}(T)$, but our experiments suggest that this variant takes considerably more time.

Example 6.6. Let $T=\left(\begin{array}{l}x \\ y\end{array}\right)^{3}$. Set $f$ and $g$ to be $\sigma_{x}(T) / T$ and $\sigma_{y}(T) / T$, respectively. Since $g$ is shift-reduced w.r.t. $y$, its kernel is equal to $g$ itself, and the corresponding shell is equal to 1 . In step 4 , we obtain $\sigma_{y}^{i}(T) \equiv\left(q_{i} / v\right) H \bmod \mathbb{U}_{H}$, where $i=0,1,2, v=(y+1)^{3}, H$ has shift quotient $g$ w.r.t. $y$, $q_{0}=\frac{1}{2}(x+1)\left(x^{2}-x+3 y(y-x+1)+1\right), \quad q_{1}=(x+1)^{3}, \quad$ and $q_{2}=\frac{(x+1)^{3}}{(x+2)^{2}}\left(11 x^{2}-12 x y+17 x+20+12 y+12 y^{2}\right)$.

By finding an $\mathbb{F}$-linear dependency among $q_{0}, q_{1}, q_{2}$, we get

$$
L:=(x+2)^{2} S_{x}^{2}-\left(7 x^{2}+21 x+16\right) S_{x}-8(x+1)^{2}
$$

is a telescoper of minimal order for $T$.

\section{IMPLEMENTATION AND TIMINGS}

We have implemented our algorithms in Maple. In order to get an idea about their efficiency, we compared their runtime and memory requirements to the performance of known algorithms. All timings are measured in seconds on a Linux computer with $388 \mathrm{~Gb}$ RAM and twelve $2.80 \mathrm{GHz}$ Dual core processors.

For the first comparison, we considered univariate hypergeometric terms of the form

$$
T=\frac{f(y)}{g_{1}(y) g_{2}(y)} \frac{\Gamma(y-\alpha)}{\Gamma(y-\beta)},
$$

where $f \in \mathbb{Z}[y]$ of degree $20, g_{i}=p_{i} \sigma_{y}^{\lambda}\left(p_{i}\right) \sigma_{y}^{\mu}\left(p_{i}\right)$ with $p_{i} \in$ $\mathbb{Z}[y]$ of degree $10, \lambda, \mu \in \mathbb{N}$, and $\alpha, \beta \in \mathbb{Z}$. For a selection of random terms of this type for different choices of $\mu$ and $\lambda$, Table 1 compares the timings of Maple's implementation of the classical Abramov-Petkovšek reduction (AP) and our modified version (MAP). We apply the algorithms to $T$ as well as to the summable terms $\sigma_{y}(T)-T$.

For the second comparison, we considered bivariate hypergeometric terms of the form

$$
T=\frac{f(x, y)}{g_{1}(x+y) g_{2}(2 x+y)} \frac{\Gamma(2 \alpha x+y)}{\Gamma(x+\alpha y)}
$$




\begin{tabular}{c|rr|rr} 
& \multicolumn{2}{|c|}{$T$} & \multicolumn{2}{c}{$\sigma_{y}(T)-T$} \\
$(\lambda, \mu)$ & AP & MAP & AP & MAP \\
\hline$(0,0)$ & 0.45 & 0.30 & 4.41 & 2.29 \\
$(5,5)$ & 5.94 & 1.21 & 10.19 & 2.40 \\
$(5,10)$ & 14.69 & 2.20 & 15.67 & 3.30 \\
$(10,10)$ & 17.22 & 2.31 & 17.98 & 2.77 \\
$(10,20)$ & 57.05 & 6.20 & 38.03 & 3.80 \\
$(10,30)$ & 316.51 & 15.73 & 74.55 & 3.73 \\
$(10,40)$ & 514.84 & 32.64 & 134.29 & 3.99 \\
\hline
\end{tabular}

Table 1: Comparison of the Abramov-Petkovšek reduction and the modified version for a collection of non-summable terms $T$ and summable terms $\sigma_{y}(T)-T$.

with $f \in \mathbb{Z}[x, y]$ of degree $n, g_{i}=p_{i} \sigma_{z}^{\lambda}\left(p_{i}\right) \sigma_{z}^{\mu}\left(p_{i}\right)$ with $p_{i} \in$ $\mathbb{Z}[z]$ of degree $m$, and $\alpha, \lambda, \mu \in \mathbb{N}$. For a selection of random terms of this type for different choices of $n, m, \alpha, \mu, \lambda$, Table 2 compares the timings of Maple's implementation of Zeilberger's algorithm $(\mathrm{Z})$ and two variants of the algorithm ReductionCT from Section 6: For the column $\mathrm{RCT}_{1}$ we computed both the telescoper and certificate, and for $\mathrm{RCT}_{2}$ we only compute the telescoper. The difference between these two variants comes mainly from the time needed to bring the rational function $u$ in the certificate $u H$ on a common denominator. When it is acceptable to keep the certificate as an unnormalized linear combination of rational functions, the timings are virtually the same as for $\mathrm{RCT}_{2}$.

\begin{tabular}{l|r|r|r|c}
$(m, n, \alpha, \lambda, \mu)$ & $\mathrm{Z}$ & $\mathrm{RCT}_{1}$ & $\mathrm{RCT}_{2}$ & order \\
\hline$(1,0,1,5,5)$ & 17.12 & 5.00 & 1.80 & 4 \\
$(1,0,2,5,5)$ & 74.91 & 26.18 & 5.87 & 6 \\
$(1,0,3,5,5)$ & 445.41 & 92.74 & 17.34 & 7 \\
$(1,8,3,5,5)$ & 649.57 & 120.88 & 23.59 & 7 \\
$(2,0,1,5,10)$ & 354.46 & 58.01 & 4.93 & 4 \\
$(2,0,2,5,10)$ & 576.31 & 363.25 & 53.15 & 6 \\
$(2,0,3,5,10)$ & 2989.18 & 1076.50 & 197.75 & 7 \\
$(2,3,3,5,10)$ & 3074.08 & 1119.26 & 223.41 & 7 \\
$(2,0,1,10,15)$ & 2148.10 & 245.07 & 11.22 & 4 \\
$(2,0,2,10,15)$ & 2036.96 & 1153.38 & 153.21 & 6 \\
$(2,0,3,10,15)$ & 11240.90 & 3932.26 & 881.12 & 7 \\
$(2,5,3,10,15)$ & 10163.30 & 3954.47 & 990.60 & 7 \\
$(3,0,1,5,10)$ & 18946.80 & 407.06 & 43.01 & 6 \\
$(3,0,2,5,10)$ & 46681.30 & 2040.21 & 465.88 & 8 \\
$(3,0,3,5,10)$ & 172939.00 & 5970.10 & 1949.71 & 9
\end{tabular}

Table 2: Comparison of Zeilberger's algorithm to reduction-based telescoping with and without construction of a certificate

\section{REFERENCES}

[1] S. A. Abramov. The rational component of the solution of a first-order linear recurrence relation with a rational right side. USSR Comp. Math. Math. Phys., 15(4):216-221, 1975.

[2] S. A. Abramov. When does Zeilberger's algorithm succeed? Adv. in Appl. Math., 30(3):424-441, 2003.

[3] S. A. Abramov and M. Petkovšek. Minimal decomposition of indefinite hypergeometric sums. In Proc. of ISSAC'01, pp. 7-14, 2001. ACM.
[4] S. A. Abramov and M. Petkovšek. Rational normal forms and minimal decompositions of hypergeometric terms. J. Symbolic Comput., 33(5):521-543, 2002.

[5] G. Almkvist and D. Zeilberger. The method of differentiating under the integral sign. J. Symbolic Comput., 10:571-591, 1990.

[6] M. Apagodu and D. Zeilberger. Multi-variable Zeilberger and Almkvist-Zeilberger algorithms and the sharpening of Wilf-Zeilberger theory. Adv. in Appl. Math., 37(2):139-152, 2006.

[7] A. Bostan, S. Chen, F. Chyzak, and Z. Li. Complexity of creative telescoping for bivariate rational functions. In Proc. of ISSAC '10, pp. 203-210, 2010. ACM.

[8] A. Bostan, S. Chen, F. Chyzak, Z. Li, and G. Xin. Hermite reduction and creative telescoping for hyperexponential functions. In Proc. of ISSAC'13, pp. 77-84, 2013. ACM.

[9] A. Bostan, P. Lairez, and B. Salvy. Creative telescoping for rational functions using the Griffith-Dwork method. In Proc. of ISSAC'13, pp. 93-100, 2013. ACM.

[10] S. Chen. Some applications of differential-difference algebra to creative telescoping. $\mathrm{PhD}$ thesis, École Polytechnique, Palaiseau, 2011.

[11] S. Chen and M. Kauers. Order-degree curves for hypergeometric creative telescoping. In Proc. of ISSAC'12, pp. 122-129, 2012. ACM.

[12] S. Chen and M. Kauers. Trading order for degree in creative telescoping. J. Symbolic Comput., 47(8):968-995, 2012.

[13] S. Chen, M. Kauers, and C. Koutschan. A generalized Apagodu-Zeilberger algorithm. In Proc. of ISSAC'14, pp. 107-114, 2014. ACM.

[14] F. Chyzak and B. Salvy. Non-commutative elimination in Ore algebras proves multivariate identities. $J$. Symbolic Comput., 26:187-227, 1998.

[15] M. C. Fasenmyer. Some generalized hypergeometric polynomials. Bull. Amer. Math. Soc., 53:806-812, 1947.

[16] R. W. Gosper, Jr. Decision procedure for indefinite hypergeometric summation. Proc. Nat. Acad. Sci. U.S.A., 75(1):40-42, 1978.

[17] M. Mohammed and D. Zeilberger. Sharp upper bounds for the orders of the recurrences output by the Zeilberger and $q$-Zeilberger algorithms. J. Symbolic Comput., 39(2):201-207, 2005.

[18] O. Ore. Sur la forme des fonctions hypergéométriques de plusieurs variables. J. Math. Pures Appl. (9), 9(4):311-326, 1930.

[19] M. Petkovšek, H. S. Wilf, and Doron Zeilberger. $A=B$. A K Peters Ltd., Wellesley, MA, 1996.

[20] M. Sato. Theory of prehomogeneous vector spaces (algebraic part). Nagoya Math. J., 120:1-34, 1990.

[21] D. Zeilberger. A fast algorithm for proving terminating hypergeometric identities. Discrete Math., 80:207-211, 1990.

[22] D. Zeilberger. A holonomic systems approach to special functions identities. J. Comput. Appl. Math., 32:321-368, 1990.

[23] D. Zeilberger. The method of creative telescoping. $J$. Symbolic Comput., 11:195-204, 1991. 